\title{
A Gas Leak and Structural Damage Detector for Spacecraft on-orbit Based on Two-staged Acoustic Sensors Array
}

\author{
Yan Rongxin*, Qi Lei \\ Vacuum and Leak Detecting Division, Beijing Institute of Spacecraft Environment Engineering, China
}

Copyright (C) 2015 by authors, all rights reserved. Authors agree that this article remains permanently open access under the terms of the Creative Commons Attribution License 4.0 International License

\begin{abstract}
The leaks are very dangerous for astronauts on orbit spacecraft. So the techniques of leak testing have been developed with acoustics method. The produce of pneumatic sound source of the leak and the gas leak detection technology based on acoustic sensor array are studied. The detection distance and direction of array are verified by the experiments. In the leak, the sound power of the gas jet is related to the diameter and length of the leak. When the measuring distance is constant, the average error of the experiment is relative to angle between leak and sensor array. When the relative angle is constant, with the increase of the distance, orientation error increases slightly, but is less than $\pm 5^{\circ}$.
\end{abstract}

\section{Keywords Spacecraft, Gas Leak, Acoustic Sensor}

\section{Introduction}

Long-term on-orbit manned spacecraft will be impacted by meteoroid and space debris, then cabin and sealing structure may damage or even perforate which can cause leakage accident ${ }^{[1]}$. So, when we continue to strengthen the ability of the reliability design of spacecraft structure, we should also enhance the ability of on-orbit leak detection, so as to take measures to ensure the reliable operation of manned spacecraft and safeguard the life safety of the astronauts. Beijing Institute of Spacecraft Environment Engineering studies the air leak testing of spacecraft cabin and carries out the research work on astronauts portable instrument which is based on ultrasonic signal ${ }^{[2-4]}$, but these instruments are not autonomous positioning. It's more urgent to study the automatic locating on-line leak detection technology which can be used on the on-orbit spacecraft and doesn't need the astronaut's operation. So the technique of the acoustic sensor have been studied to localize the leaks on orbit spacecraft automatically. It is necessary to understand the relation between the leaks on spacecraft and acoustic signal.

\section{Principle Analysis}

\subsection{Leak Causes Acoustic Signal}

The gas leak out along the channel under the action of the pressure difference. When gas passes through the micro aperture, it is fast and in a turbulent state, and contains a large number of turbulent eddies. The internal stress of gas turbulence eddies makes the respective volume elements suffer from equal and opposite fluctuating force, which causes that gas produces quadrupole sound jet. For the leak whose diameter is $d$, length is $l$, the sound power at the jet is ${ }^{[5]}$ :

$$
p_{T} \propto \frac{\rho_{0}(u d / l)^{8} l}{c_{0}^{5}}\left(\frac{\rho_{s}}{\rho_{0}}\right)^{2}
$$

In the equation, $C_{0}$ is velocity of wave propagation in the atmosphere in cabin, $\rho_{0}$ is the atmospheric density in cabin, $\rho_{s}$ is standard atmospheric density, $u$ is velocity of gas jet at nozzle. For the hermetically sealed cabin of the manned spacecraft, pressure will change for the leak, the pressure $p_{t}$ is:

$$
p_{\mathrm{t}}=\left(p_{0}-p_{\text {out }}\right) e\left(-\frac{C}{V} t\right)+p_{\text {out }}
$$

In the equation, $c$ is the conductance of the leak, $V$ is the volume of the cabin, $p_{0}$ is the initial pressure of the cabin, $p_{\text {out }}$ is the pressure of the external vacuum environment. In general, $\mathrm{V}$ is the volume of the cabin of the manned spacecraft from $10 \mathrm{~m}^{3}$ to $100 \mathrm{~m}^{3}$. The cabin's possible leak diameter is $0.1 \mathrm{~mm}$ to $2 \mathrm{~mm}$. According to equation (2), for the leak which is $0.1 \mathrm{~mm}$ in the cabin that is $10 \mathrm{~m}^{3}$, even make 
the gas pressure drop $1 \mathrm{kPa}$, It needs $10.5 \mathrm{~h}$. When the time of leak detection is several minutes, $p_{t}$ change just a little. The pressure of the exit is in vacuum, the flow velocity ${ }^{[6]}$ is:

$$
u=\sqrt{\frac{2 \gamma}{\gamma-1} \frac{p_{t}}{\rho_{0}}\left[1-\left(\frac{p_{\text {out }}}{p_{t}}\right)^{\frac{\gamma-1}{\gamma}}\right]}
$$

In the equation, $u$ is the flow velocity of gas molecule in the leak. The equation (3) to equation (1):

$$
P_{\mathrm{T}} \propto \frac{\left\{\frac{2 \gamma}{\gamma-1} \frac{p_{\mathrm{t}}}{\rho_{0}}\left[1-\left(\frac{p_{\text {out }}}{p_{t}}\right)^{\frac{\gamma-1}{\gamma}}\right]\right\}^{4} d^{8} \rho_{s}^{2}}{\rho_{0} c_{0}^{5} l^{7}}
$$

As for the leak of large cabin, when the time for leak detection is several minutes, all change a little, according to (4), we can know: in the leak, the sound power of the gas jet is decided by the diameter and length of the leak. when the length of the leak is constant, the greater the diameter is, the greater the sound power is. So if we can determine the sound power in the leak, we can determine the geometry and the leak rate of the leak.
The acoustic signal produced by the leak from the atmosphere in the spacecraft to the vacuum side will be transmitted to piezoelectric acoustic emission sensor which is coupled on the outer wall of the structure. The acoustic signal is converted to electrical signal, and we carry out the leakage characteristic analysis of the acoustic emission signals collected. We can use acoustic emission technology to detect the leakage phenomena from the atmosphere to the vacuum side and estimate the information of the leak.

\subsection{Detection of Acoustical Signal Caused by Leak}

Hardware part of the detection system is composed of the leak simulation device, bulkhead breadboard for on-orbit spacecraft and the acquisition unit for the leak acoustical emission signal. Figure 1 indicates the overall structure. The square formed by solid lines in the picture represents the bulkhead breadboard for the on-orbit spacecraft. The circle in the center of the breadboard represents the vacuum-sealed cylinder. Rectangle numbered from 1 to 8 represents eight pre-amplifiers.
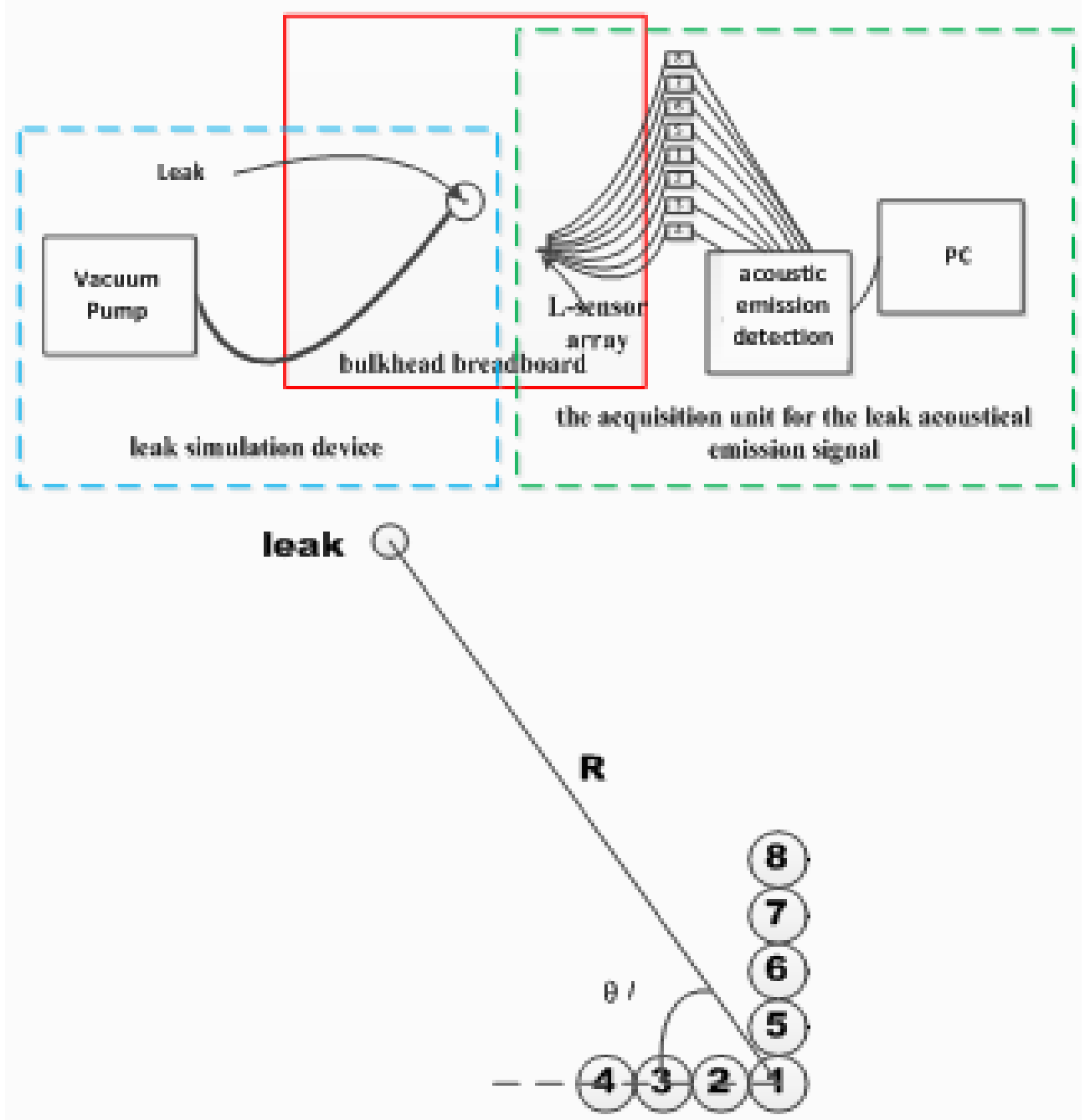

Figure 1. Detection system of leak acoustical emission signal 
According to Beam Forming algorithm principle, the test takes sensor No.1 as the reference and delays the signals of sensors No.2 to No.8, then takes superposition of the eight signals. Assume that the differences between the distances from the sensors No.2 till No.8 to the signal source with that of No.1 as $d_{2}, d_{3} d_{4} \ldots d_{8}$, and that the relative angle between the leak source and sensor array as $\theta^{\prime}$ and the diameter of sensor as D. Then we have relations as follow:

$$
\begin{aligned}
& d_{i}=(i-1) D \cdot \cos \theta^{\prime} \quad(i=1,2,3,4) \\
& d_{i}=(i-1) D \cdot \sin \theta^{\prime} \quad(i=5,6,7,8)
\end{aligned}
$$

While the acoustical signals spread with speed $\boldsymbol{V}$, the delay from the signals of sensor No.2 to No.8 to that of sensor No. 1 is:

$$
\mathrm{t}_{i}=\frac{d_{i}}{v}(i=2,3,4,5,6,7,8)
$$

Take the superposition of the delay signals from sensors No.1 to No.8, then we have:

$$
\Psi_{\Sigma}=\sum_{\mathrm{i}=1}^{8} \Psi\left(\mathrm{t}+\mathrm{t}_{\mathrm{i}}\right)=\sum_{\mathrm{i}=1}^{8} \Psi\left(\mathrm{t}+\frac{\mathrm{d}_{\mathrm{i}}}{\mathrm{v}}\right)
$$

Then the energy of the eight signals after superposition is:

$$
\mathrm{P}=\sum_{\mathrm{i}=1}^{8} \Psi^{2}\left(\mathrm{t}+\mathrm{t}_{\mathrm{i}}\right)=\sum_{\mathrm{i}=1}^{8} \Psi^{2}\left(\mathrm{t}+\frac{\mathrm{d}_{\mathrm{i}}}{\mathrm{v}}\right)
$$

The speed $v$ is a constant while conducting one positioning calculating, so $P$ only remains the function of positioning angle $\boldsymbol{\theta}$. When $\mathrm{P}$ reaches its maximum, the corresponding $\theta$ indicates the direction of the leak source. But wave velocity $\boldsymbol{V}$ varies with the change of the frequency. The energy of different components is $P_{1}, P_{2}, P_{3, \ldots} P_{8}$, the corresponding weight are $Q_{1}, Q_{2}, Q_{3}, \ldots Q_{8}$. Define $P$ as the energy get after normalization of the energy components. $P$ is calculated by the follow formula:

$$
\mathrm{P}=\sum_{\mathrm{i}=1}^{\mathrm{n}} \mathrm{Q}_{\mathrm{i}} \cdot \frac{\mathrm{P}_{\mathrm{i}}}{\mathrm{P}_{\mathrm{i}}(\max )} \times \frac{1}{\sum_{\mathrm{i}=1}^{8} \mathrm{Q}_{\mathrm{i}}}
$$

In the equation $(9), \operatorname{Pi}(\max )$ is the energy relative to the peak values in the Energy-Angle figure of the components.

\section{Test Result}

\subsection{Test for the Effect of Relative Angle between Leak and Sensor Array to the Directional Accuracy}

In order to research the effect of relative angle between leak and sensors to the directional accuracy, the test choose the leak signals of coordinate $(0,0)$ as the object. Define the angle between the line connected the tested leak with sensor No.1 and the short edge of the sensor array (edge formed by sensors No.1 to No.4) as the relative angle $\theta$ between leak and sensor array. Define the distance from the center of sensor No. 1 in the sensor array to the tested leak as R. Figure 2 is the scatter diagram of the average error. Concluded from Figure 2 the average error reaches the minimum at $\theta=55^{\circ}$.

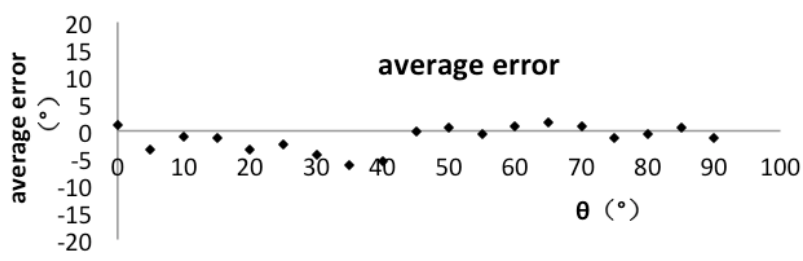

Figure 2. the scatter diagram of positioning error and $\theta, R=30 \mathrm{~cm}$

\subsection{Test about the Effect of the Distance between the Leak and Sensors to the Positioning Accuracy}

One of the factors that affect the positioning accuracy is the distance between the leak and the center of sensor array $\mathrm{R}$. In order to research the effect of $\mathrm{R}$ to the positioning accuracy, the test ranges $\mathrm{R}$ as far as possible. So keep the angle to the leak remaining $45^{\circ}$ unchanged, the test starts from $R=1 \mathrm{~cm}$, stepped by $1 \mathrm{~cm}$.

Figure 3 indicates the positioning result when the angle is $45^{\circ}$. It can be concluded that with the increase of the distance, the positioning error increases slightly while all the errors smaller than $\pm 5^{\circ}$.

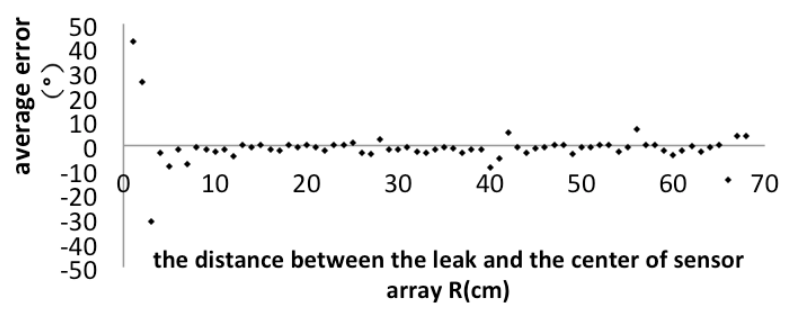

Figure 3. the average positioning error of positioning accuracy caused by $\mathrm{R}$

\section{Conclusions}

We study the formation of pneumatic sound source of the leak which is caused in the manned spacecraft with large cabin by the space debris, and acoustic emission signal beam-forming positioning mechanism, and the gas leak detection technology which is based on acoustic sensor array, and then verify the detection distance and direction of array by the experiments, the main conclusions are as follows:

(1) In the leak, the sound power of the gas jet is related to the diameter and length of the leak, when the length of the leak is constant, the greater the diameter is, the greater the sound power is. If we can determine the sound power or energy in the leak, we can determine the geometry and the leak rate of the leak.

(2) When the measuring distance is constant, if the relative angle between leak and sensor array is $55^{\circ}$, the average error of the experiment is minimum. 
(3) When the relative angle between leak and sensor array is constant, with the increase of the distance, orientation error increases slightly, but is less than $\pm 5^{\circ}$.

\section{REFERENCES}

[1] Yan Lie Deng, Ningfeng. Translator Accident and policy for Spacecraft system[M]. Chinese Spacecraft Press 2007.

[2] Yan Rongxin, Li Weidan. Study on the leak test technology of spacecraft using ultrasonic[C]. $2^{\text {th }}$ Singapore International
Non Destructive Testing Conference and Exhibition. Singapore. June.19-21. 2013.

[3] Yan Rongxin. Design of portable leak detector used for manned spacecraft based on ultrasonic[C]. 11 ${ }^{\text {th }}$ European Conference Non-destructive. Prague . May 6-9, 2014.

[4] Yan Rongxin, Qi Lei. A gas leak and structural damage detector for spacecraft on orbit based on two staged acoustic sensors array [C]. 2015 International Congress on Ultrasonics. Metz France. May 11-14. 2015.

[5] Zhangqiang, Pneumatic Acoustics[M], Beijing: National Defense Industry Press, 2012: 210-211.

[6] Chen Zhouru, Jin Chaoming. Hydrodynamics [M], Beijing Higher Education Press 2004: 410-411 